\title{
Effect of microRNA-21 on multidrug resistance reversal in A549/DDP human lung cancer cells
}

\author{
ZUOLIANG DONG ${ }^{1 *}$, LI REN $^{2 *}$, LI LIN $^{3}$, JIANG LI $^{1}$, YIWEN HUANG $^{1}$ and JINHONG LI \\ ${ }^{1}$ Department of Clinical Laboratory, Tianjin Medical University General Hospital, Heping District, Tianjin 300052; \\ ${ }^{2}$ Department of Clinical Laboratory, Key Laboratory of Cancer Prevention and Therapy; ${ }^{3}$ Department of Thoracic Oncology, \\ Tianjin Lung Cancer Center, Tianjin Medical University Cancer Institute and Hospital, Tianjin, Hexi 300060, P.R. China
}

Received November 22, 2013; Accepted July 9, 2014

DOI: $10.3892 / \mathrm{mmr} .2014 .2662$

\begin{abstract}
Lung cancer is a predominant cause of cancer-related mortality and numerous lung cancer patients succumb to the disease due to drug resistance. A number of microRNAs (miRNAs) are upregulated in cancer and are involved in tumorigenesis, functioning as oncogenes. Several functional studies have shown that miR-21 is important in carcinogenesis; however, none of these studies has investigated multidrug resistance (MDR) reversal in human lung cancer cells. In the present study, the effect of miR-21 on MDR reversal was analyzed in A549/DDP lung cancer cells. The data demonstrated the following after miR-21 silencing: Proliferation of the tumor cells was inhibited, cell apoptosis and oxidative damage were increased, the cell cycle was blocked at the G0/G1 phase, expression levels of P-glycoprotein were reduced, accumulation of Rhodamine 123 was increased, and the MDR-related genes encoding MDR1, MPR, glutathione S-transferase- $\pi$, B-cell lymphoma 2, cyclin-dependent kinase 1, cystathione and glutathione were downregulated. Further mechanistic analysis revealed that miR-21 silencing reduced AKT phosphorylation and transcriptional activation of E2F-1 and Twist. In conclusion, this study demonstrated that miR-21 silencing reversed lung cancer cell MDR by modulation of MDR-related gene expression and inhibition of the AKT signaling pathway, suggesting that miR-21 may be a potential therapeutic candidate in patients with MDR lung cancer.
\end{abstract}

\section{Introduction}

MicroRNA (miRNA) molecules have gradually become a key area in biomedical research since the identification of the first

Correspondence to: Mr. Zuoliang Dong, Department of Clinical Laboratory, Tianjin Medical University General Hospital, 154 Anshan Road, Heping District, Tianjin 300052, P.R. China E-mail: zuoliang_dong77@126.com

*Contributed equally

Key words: microRNA-21, multidrug resistance, lung cancer type of miRNA molecule, lin-4, in nematodes by Lee et al using genetic analyses (1). miRNAs are endogenous single-stranded non-coding small RNA molecules of length 22 basic groups; this type of RNA is evolutionarily conserved in terms of the genetic features. miRNAs are located in genomes as single copies, multiple copies or gene clusters. The majority are situated in intergenic sequences; a few miRNAs are also located in intronic regions, which are transcribed together with host genes in the form of polycistrons $(2,3)$.

As non-coding small molecule RNAs involved in post-transcriptional regulation, miRNAs can regulate gene expression by binding with the bases of the 3'-non-coding region of messenger RNA through the following three approaches: Forming full complementation with the messenger RNA and inducing the endonuclease effect; forming partial complementation with the messenger RNA and thus affecting translation; and forming partial complementation with the messenger RNA and thus inducing deadenylation and degradation. Previous research has suggested that miRNAs, a newly identified gene regulation subfamily, are involved in various biological processes, including cell proliferation, differentiation, apoptosis, stress tolerance and metabolic activities in various types of tissue. In recent years, the involvement of miRNAs in the occurrence, development and metastasis of malignant tumors has drawn wide attention (4-6). Previous studies have indicated that $50 \%$ miRNAs are located in either genomic regions associated with tumor regulation, fragile positions of the chromosome or certain tumor-related genomic sequences. Other studies have indicated that miRNAs are key to the occurrence of tumors, mainly through the following: A section of the miRNA-encoded gene may act as an oncogene; a section of the miRNAs-encoded gene may act as an anti-oncogene; and certain oncogene-encoded miRNA genes may be involved in the occurrence of associated tumors (7). Following analysis, the miRNA expression levels in various human tumor types were also determined to be markedly different. For example, the expression levels of let7, miR15 and miR16 were reduced in patients with lung cancer and leukemia, whereas the expression levels of miR155, miR211 and miR31 were found to be increased in certain types of tumor tissue and cell lines $(8,9)$.

miR-21 is one of the most commonly investigated miRNAs in cancer-related research (10). The expression levels of miR-21 are increased in numerous types of tumor, and are closely associ- 
ated with the occurrence, development, metastasis and prognosis of a variety of cancer types, including breast, prostate, lung and stomach cancers, glioblastoma, cholangiocarcinoma and pancreatic endocrine tumors (11-14). A recent study has also shown that miR-21 is over-expressed in lung tumors (15).

The tumor cells in which chemotherapy fails commonly exhibit multidrug resistance (MDR). MDR refers to the generation of resistance in malignant cells to a variety of antitumor drugs with distinct structures and functional mechanisms, subsequent to contact with one type of antitumor drug. MDR often results in failure of cancer chemotherapy and clinical recurrence of the cancer. In MDR, tumor cells undergo self-adaptation through contact with chemotherapeutic drugs (16). The generation of MDR involves numerous concurrent molecular mechanisms, including: The increase of drug efflux molecules expressed on the membrane, for example, P-glycoprotein (P-gp), MDR-related protein and lung resistance-related protein reduce drug absorption while increasing drug efflux; the enhancement of cellular detoxification capability, for example, changes in the activity and expression of glutathione S-transferase- $\pi$ (GST- $\pi$ ), and alterations in metallothionein expression; modification of DNA repair capacity, for example, changes in O6-methylguanine-DNA methyltransferase activity; alterations in intra-cellular proteins, such as the variations in topoisomerase activity and expression; changes in the expression levels and activity of protein kinase $\mathrm{C}$ and various other isozymes; and the inhibition of apoptosis (17-20).

In view of the important role of miR-21 in tumors, miR-21 may be key in cisplatin resistance in lung cancer. Therefore, the present study examined the effect of miR-21 on the reversal of cisplatin resistance in A549/DDP cells and investigated the associated mechanism.

\section{Materials and methods}

Cell lines and main reagents. The A549 and A549/DDP human lung cancer cell lines used in this study were obtained from the China Center for Type Culture Collection (Wuhan, China); Dulbecco's modified Eagle's medium was purchased from Gibco-BRL (Carlsbad, CA, USA); the miR-21 inhibitor and miR-21 control were bought from Shanghai GenePharma Co, Ltd. (Shanghai, China); the transfection reagent Lipofectamine $^{\mathrm{TM}} 2000$ and Opti MEM $^{\circledR}$ were purchased from Invitrogen Life Technologies (Carlsbad, CA, USA); the TaqMan miRNA Isolation kit, the TaqMan miRNA Assay and the TaqMan Universal polymerase chain reaction (PCR) Master mix were supplied by Applied Biosystems (Carlsbad, CA, USA); and the SYBR ${ }^{\circledR}$ Green Real-time PCR Master mix was purchased from Takara Bio, Inc. (Shiga, Japan). All the antibodies were purchased from Santa Cruz Biotechnology, Inc. (Santa Cruz, CA, USA), with the exception of antibodies against $\beta$-actin, which were obtained from Sigma-Aldrich (St. Louis, MO, USA).

Cell culture. The cells were cultured in RPMI-1640 medium, with added $10 \%$ fetal bovine serum, $100 \mathrm{U} / \mathrm{ml}$ penicillin and $100 \mathrm{U} / \mathrm{ml}$ streptomycin, at $37^{\circ} \mathrm{C}, 5 \% \mathrm{CO}_{2}$ and saturated humidity. Cross-breeding was performed using trypsin once every two days. The cells were extracted in the logarithmic growth phase for subsequent experiments.
Antisense oligonucleotide transfection assay. The miR-21 inhibitor and the miR-21 control plasmids were transfected using Lipofectamine $^{\mathrm{TM}}$ 2000. Briefly, the miR-21 inhibitor and miR-21 control were diluted and mixed with serum-free Opti-MEM ${ }^{\circledR}$ medium at room temperature for $5 \mathrm{~min}$. Subsequently, Lipofectamine $^{\mathrm{TM}} 2000$ was mixed with the solution, which was incubated at room temperature for $20 \mathrm{~min}$. A549/DDP cells were transfected with the miR-21 inhibitor and the miR-21 control, respectively. The A549/DDP cells transfected with miR-21 inhibitor were designated the ASO-miR-21 group, the A549/DDP cells transfected with miR-21 control were identified as the negative group (ASO-miR-NC), A549/DDP cells without treatment were classified as the control group and A549 cells were termed the parent group.

miR-21 detection assay. Small RNA was extracted from cells in each group using the TaqMan miRNA Isolation kit. TaqMan miRNA assay reagents and TaqMan Universal PCR Master mix were used to detect the expression levels of mature miR-21. U6 served as the reference gene.

Cell viability detection assay. A total of $3 \times 10^{5}$ cells in each experimental condition group were cultured in a 6-well plate for $24 \mathrm{~h}$, then $0,1,2,5,10,20,50$ or $100 \mu \mathrm{M}$ cisplatin was added for a further $72 \mathrm{~h}$ incubation at $37^{\circ} \mathrm{C}$. Cell viability was determined by a Vi-CELL Cell Viability Analyzer (Beckman Coulter, Brea, CA, USA), according to the manufacturer's instructions and the cell proliferation inhibition rate was calculated.

Apoptosis and cell cycle assays. Apoptosis was measured using Annexin V-fluorescein isothiocyanate (FITC) and propidium iodide (PI; BD Pharmigen, San Diego, CA, USA). Briefly, $3 \times 10^{5}$ cells from each group were seeded in 6-well plates for $24 \mathrm{~h}$, then 5 or $10 \mu \mathrm{M}$ cisplatin was added for a further $24 \mathrm{~h}$ incubation. The cells were trypsinized, washed once with phosphate-buffered saline (PBS) and stained with FITC-labeled anti-Annexin V antibody and PI for $15 \mathrm{~min}$ in the dark. The fluorescence was assessed by BD FACS flow cytometry (BD Biosciences, Franklin Lakes, NJ, USA).

To analyze the cell cycle, a total of $3 \times 10^{5}$ cells from each group were seeded in 6-well plates for $24 \mathrm{~h}$, then the cells were trypsinized, washed once with PBS and stained with ice-alcohol overnight at $4^{\circ} \mathrm{C}$. RNase A and PI were added and the cells were incubated at $37^{\circ} \mathrm{C}$ for $30 \mathrm{~min}$. Fluorescence was assessed by BD FACS flow cytometry.

Rhodamine 123 accumulation and P-glycoprotein (P-gp) expression assay. Rhodamine 123 accumulation and $\mathrm{P}-\mathrm{gp}$ expression levels were measured to evaluate MDR in the cancer cells. Briefly, to analyze Rhodamine 123 accumulation, $3 \times 10^{5}$ cells from each treatment group were seeded in 6-well plates for $24 \mathrm{~h}$. The cells were then trypsinized, suspended and incubated in phenol-red-free minimum essential medium containing $10 \mu \mathrm{g} / \mathrm{ml}$ Rhodamine 123 (Sigma-Aldrich) for $20 \mathrm{~min}$ at $37^{\circ} \mathrm{C}$. Following incubation, the cell monolayers were washed three times with ice-cold PBS and the fluorescence was measured by BD FACS flow cytometry at an excitation wavelength of $485 \mathrm{~nm}$ and an emission wavelength of $535 \mathrm{~nm}$. 
For the P-gp expression assay, a total of $3 \times 10^{5}$ cells from each group were seeded in 6-well plates for $24 \mathrm{~h}$. The cells were then trypsinized, resuspended and incubated with phycoerythrin (PE)-P-gp, for $30 \mathrm{~min}$ at $37^{\circ} \mathrm{C}$. Following incubation, the cell monolayers were washed three times with ice-cold PBS and the fluorescence was determined by BD FACS flow cytometry at an excitation wavelength of $485 \mathrm{~nm}$ and emission wavelength of $535 \mathrm{~nm}$.

Oxidative damage detection assay. Cellular oxidative damage was measured using a reactive oxygen detection kit (Beyotime Biotech, Jiangsu, China). Briefly, $3 \times 10^{5}$ cells from each treatment group were seeded in 6 -well plates for $24 \mathrm{~h}$, then 5 or $10 \mu \mathrm{M}$ cisplatin was added for another $24 \mathrm{~h}$. The cells were trypsinized, washed once with PBS and stained with dichloro-dihydro-fluorescein diacetate (10 $\mu \mathrm{mol} / \mathrm{l}$; Beyotime, Shanghai, China) for $30 \mathrm{~min}$ in the dark. Fluorescence was detected by BD FACS flow cytometry.

Cystathione and glutathione (GSH) detection assay. A total of $3 \times 10^{5}$ cells in each experimental group were seeded in 6-well plates for $24 \mathrm{~h}$. The cells were then trypsinized, resuspended, and the quantities of cystathione and GSH were detected according to the instructions of the respective assay kits provided by Nanjing Jiancheng Bioengineering Institute (Nanjing, China).

MDR gene mRNA and protein expression assays. The protein expression levels of the MDR genes were measured by western blotting. A total of $3 \times 10^{5}$ cells were seeded in 6-well plates for $24 \mathrm{~h}$ and lysed in lysis buffer containing phenylmethylsulfonyl fluoride and EGTA (Beyotime). Total protein was extracted and quantified colorimetrically by the bicinchoninic acid method (Beyotime). Protein (100 $\mu \mathrm{g}$ ) was separated by $10 \%$ SDS-PAGE electrophoresis and the samples were transferred to a polyvinylidene difluoride membrane. The membrane was blocked in Tris-buffered saline containing Tween 20 (TBST) and 5\% skimmed milk powder for $2 \mathrm{~h}$ at $37^{\circ} \mathrm{C}$. The respective antibodies against B-cell lymphoma 2 (BCL-2; 1:800), cyclin-dependent kinase 1 (CDK1; 1:500), GST- $\pi$ (1:800), MDR1 (1:800), MPR1 (1:800), p-AKT (1:300) and $\beta$-actin $(1: 5,000)$ were added to the samples, which were incubated overnight at $4^{\circ} \mathrm{C}$. The membranes were washed three times with TBST buffer for 10 min each. Rabbit anti-mouse $\operatorname{IgG}$ (1:1,000 diluted) labeled with horseradish peroxidase enzyme was added to the samples, which were incubated for $2 \mathrm{~h}$ at $37^{\circ} \mathrm{C}$. The bands were then detected with enhanced chemiluminescence(EMD Millipore, Billerica, MA, USA). $\beta$-actin served as the internal reference.

The mRNA expression levels of the MDR genes were measured by quantitative PCR assay. Firstly, a total of $3 \times 10^{5}$ cells were seeded in 6 -well plates for $24 \mathrm{~h}$ and $80 \mathrm{ng}$ total RNA was reverse-transcribed with an RNA PCR kit (Takara Bio, Inc., Shiga, Japan), which was subsequently quantified with the SYBR ${ }^{\circledR}$ Green Real-Time PCR Master mix kit (Takara Bio, Inc.). The primer sequences of the genes were the following: BCL-2 forward, 5'-GGTGAACTGGGGGAGGATTGT-3' and reverse, 5'-CTTCAGAGACAGCCAGGAGAA-3'; CDK1 forward, 5'-GATTCTATCCCTCCTGGT-3' and reverse, 5'-TAGGCTTCCTGGTTTCC-3'; GST- $\pi$ forward, 5'-GGACCTCCGCTGCAAATAC-3' and reverse, 5'-GAAGGTCTTGCCTCCCTGG -3'; MDR1 forward,
5'-AAAAAGATCAACTCGTACCACTC-3' and reverse, 5'-GCACAAAATACACCAACAA-3'; MPR1 forward, 5'-ACTTCCACATCTGCTTCGTCAGTG-3' and reverse, 5'-ATTCAGCCACAGGAGGTAGAGAGC-3'; GAPDH forward, 5'-AAGGTGAAGGTCGGAGTCAA-3' and reverse, 5'-AAGTGGTCGTTGAGGGCAAT-3'. GAPDH served as an internal control. Using an ABI 7500 real time PCR system (Applied Biosystems China, Beijing, China), the reaction conditions were: $30 \mathrm{sec}$ at $95^{\circ} \mathrm{C}, 60 \mathrm{sec}$ at $65^{\circ} \mathrm{C}$ and $60 \mathrm{sec}$ at $72^{\circ} \mathrm{C}$ for 40 cycles, then the reaction was terminated at $4^{\circ} \mathrm{C}$.

Dual luciferase assay. The cells were transfected with the miR-21 inhibitor and the miR-21 control, respectively, in 24-well plates with $400 \mathrm{ng}$ E2F-1, Twist luciferase reporter and 10 ng Renilla reporter for $24 \mathrm{~h}$. The lysates were collected and the fluorescence intensity was measured using a dual luciferase reporter system (Promega Corporation, Madison, WI, USA) in 96-well plates.

Statistical analysis. Data are presented as the means \pm standard deviation and were analysed by SPSS 11.0 software (SPSS, Inc., Chicago, IL, USA). Statistical analyses were performed using one-way analysis of variance. $\mathrm{P}<0.05$ was considered to indicate a statistically significant difference.

\section{Results}

miR-21 expression levels are increased in A549/DDP cisplatin-resistant lung cancer cells. The expression levels of miR-21 were detected in A549 lung cancer cell lines and in A549/DDP cisplatin-resistant cell lines. The results revealed that the expression of miR-21 was significantly upregulated in A549/DDP cells as compared with the A549 cells $(\mathrm{P}<0.05)$. Whether miR-21 is important in cisplatin resistance was also analyzed in view of its role in cancer promotion. miR-21 expression in A549/DDP cells was knocked down by antisense oligodeoxynucleotides (ASO) to establish A549/DDP miR-21 silencing cell lines (ASO-miR-21-1 group), which exhibited significantly reduced miR-21 expression levels, as compared with the control group $(\mathrm{P}<0.05 ;$ Fig. 1).

Cisplatin resistance is reversed following miR-21 silencing. Subsequently, cisplatin sensitivity was examined in A549, A549/DDP and A549/DDP cells following miR-21 knockdown. As shown in Fig. 2, subsequent to knockdown of miR-21 expression by ASOs, the cisplatin sensitivity of A549/DDP cells was significantly increased after $72 \mathrm{~h}$ cisplatin treatment, as detected by the cell viability detection assay. The half maximal inhibitory concentration $\left(\mathrm{IC}_{50}\right)$ of the ASO-miR-21 group was $22.2 \mu \mathrm{M}$ and the $\mathrm{IC}_{50}$ of the control group was $48.6 \mu \mathrm{M}$, with reversal fold change 2.19. These results suggest that miR-21 is important in the generation of cisplatin resistance in tumors.

miR-21 increases cisplatin resistance in tumor cells by inhibiting apoptosis and blocking the cell cycle. Cisplatin exerts a tumor suppressive effect mainly by promoting the apoptosis of tumor cells. Therefore, the impact of miR-21 on cisplatin-mediated apoptosis was examined. Subsequent to miR-21 expression knockdown, the apoptotic rate in A549/DDP cells was significantly increased $(\mathrm{P}<0.05$; Fig. $3 \mathrm{~A})$, while the expres- 


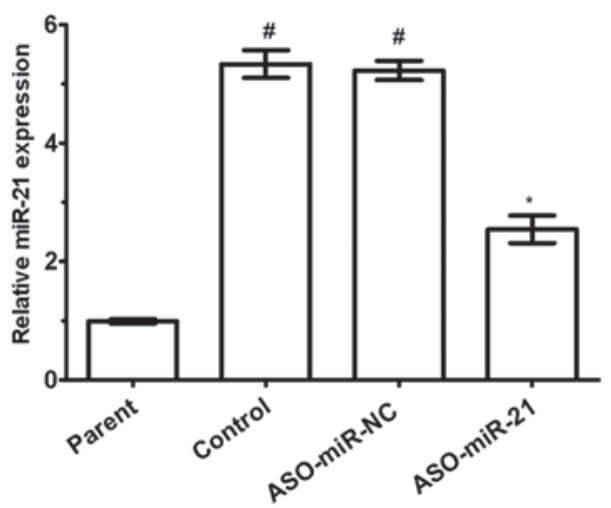

Figure 1. Expression levels of miR-21 in human lung cancer cell lines. The quantitative polymerase chain reaction results showed that miR-21 expression was significantly upregulated in A549/DDP cells as compared with A549 cells. Reduced miR-21 expression levels in the miR-21-silencing A549/DDP group (ASO-miR-21 cells) were detected as compared with the positive (A549/DDP cells) and negative control (ASO-miR-NC cells) groups. The data are presented as the means \pm standard deviation. ${ }^{~} \mathrm{P}<0.05$, as compared with the parent group; ${ }^{~} \mathrm{P}<0.05$, as compared with the control group; $\mathrm{n}=5$. miR-21, microRNA-21; ASO, antisense oligonucleotides; NC, negative control.

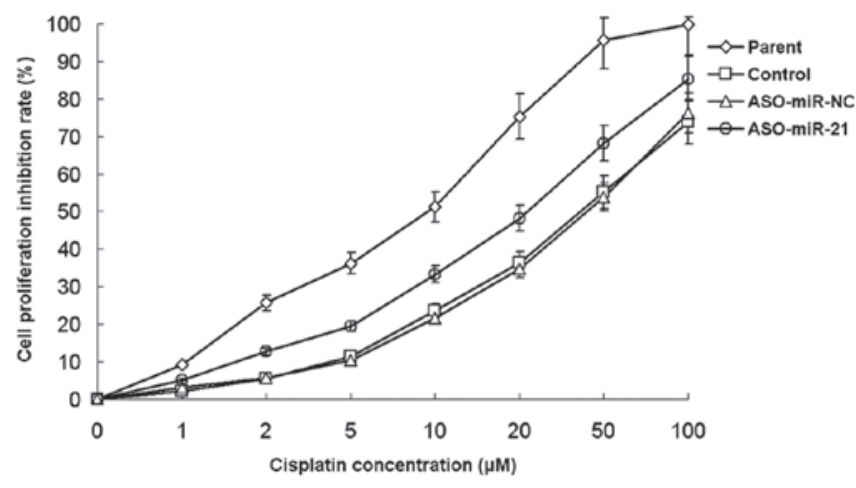

Figure 2. miR-21 silencing reverses cisplatin resistance in A549/DDP human lung cancer cell lines. The Vi-CELL Cell Viability Analyzer results showed an increased sensitivity to cisplatin in the miR-21-silencing A549/DDP group (ASO-miR-21 cells), as compared with the positive (A549/DDP cells) and the negative control (ASO-miR-NC cells) groups. The data are presented as the means \pm standard deviation. $\mathrm{n}=10$. miR-21, microRNA-21; ASO, antisense oligonucleotides; $\mathrm{NC}$, negative control.

sion levels of the apoptosis suppression protein BCL-2 were significantly reduced ( $\mathrm{P}<0.05$; Fig. $3 \mathrm{C})$, as compared with the control group. This suggests that miR-21 induces cisplatin resistance by inhibiting apoptosis. Simultaneously, the cell cycle of A549/DDP cells was arrested at the G0/G1 phase (Fig. 3B) and CDK1 expression levels were significantly reduced in the miR-21 knockdown cells, as compared with the control cells $(\mathrm{P}<0.05$; Fig. $3 \mathrm{C})$. This indicates that miR-21 also induces cisplatin resistance by blocking the cell cycle.

miR-21 increases cisplatin resistance by drug excretion. The increase in cell efflux is an important mechanism in the generation of MDR and a common cause of cisplatin resistance. Thus, Rhodamine 123 accumulation and P-gp expression levels were examined. As determined by the flow cytometry results, Rhodamine 123 accumulation was significantly increased in A549/DDP cells following miR-21 silencing (Fig. 4A), in comparison with the control cells, showing that the cell efflux capacity was reduced. The P-gp expression levels were significantly reduced (Fig. 4B). This indicated that miR-21 may be important in enhancing cell efflux. These results suggest that miR-21 enhances cell efflux by increasing P-gp expression levels, which in turn reduces the cell sensitivity to cisplatin.
miR-21 increases cisplatin resistance by inhibiting cell oxidative damage metabolism. The induction of apoptosis through increased cell oxidative damage is one mechanism by which cisplatin causes tumor cell death. By analyzing the relative ROS expression levels in the different groups, increased oxidative damage was identified in the miR-21-silencing A549/DDP cells (Fig. 5A). Furthermore, the expression levels of cystathione and GSH following knockdown of miR-21 expression were examined. As shown in Fig. 5B, the expression levels of cystathione and GSH in A549/DDP cells were significantly reduced following knockdown of miR-21 expression $(\mathrm{P}<0.05)$. These results suggest that miR-21 is important in inhibiting cisplatin-mediated apoptosis by increasing cell cystathione and GSH expression levels.

miR-21 enhances MDR gene expression levels in cells. GST- $\pi$ is an important cell detoxifying enzyme, and MDR1 and MPR1 are important drug resistance genes that produce an excavating pump effect. Increased expression levels of these genes is a common cause of drug resistance. Following knockdown of miR-21 expression, the GST- $\pi$, MDR1 and MPR1 protein expression levels were reduced. The mRNA expression levels of these genes were also significantly reduced $(\mathrm{P}<0.05$; Fig. 6$)$. 
A
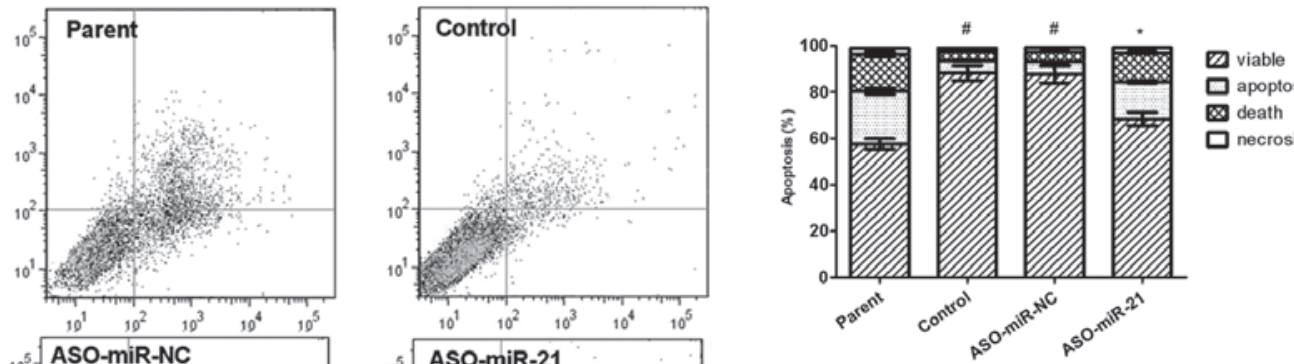

口 apoptosis

death
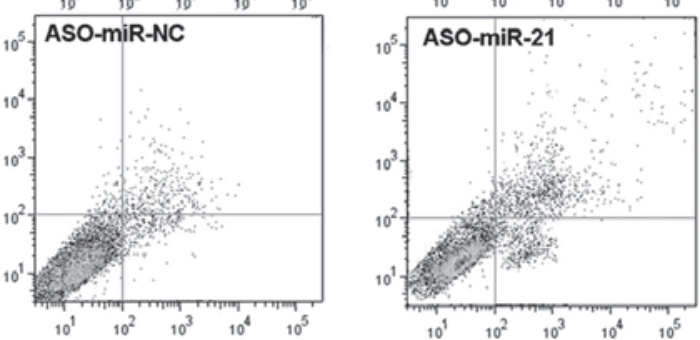

口 necrosis

B
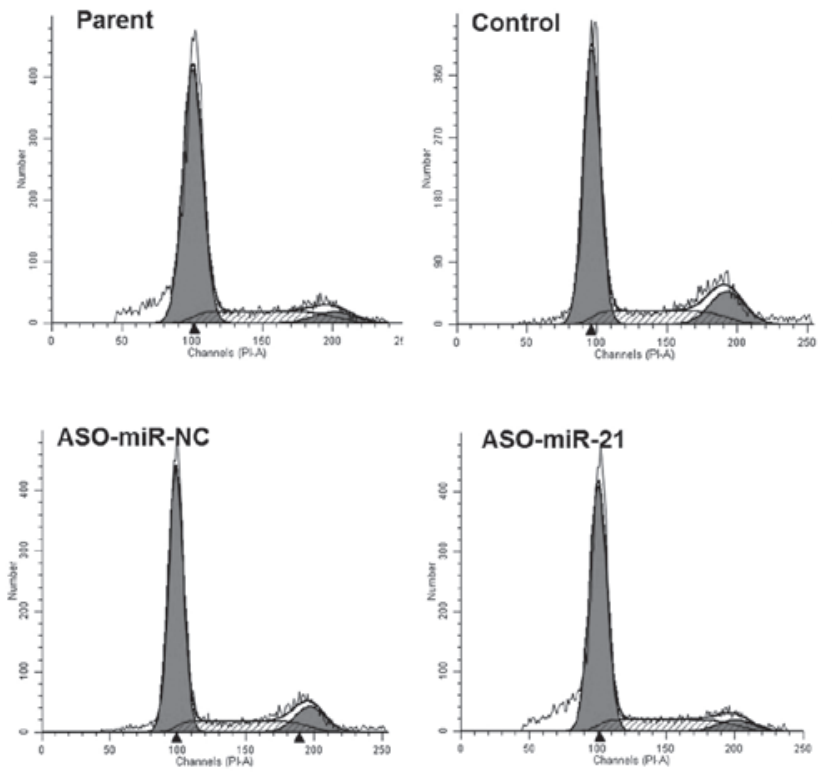
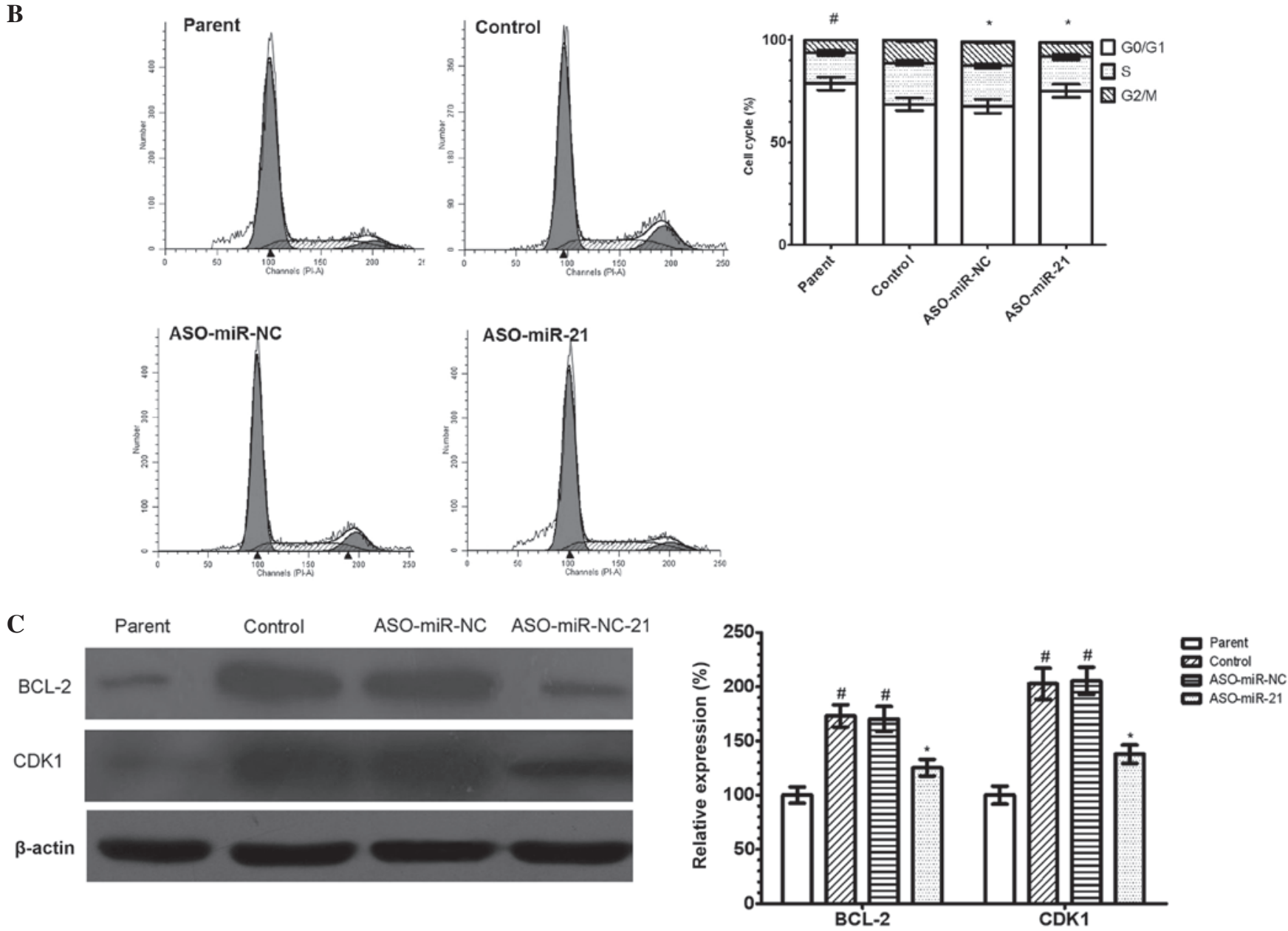

Figure 3. miR-21 increases cisplatin resistance in human lung cancer cells by inhibiting apoptosis and blocking the cell cycle. (A) Flow cytometric results showed increased apoptosis in the miR-21-silencing A549/DDP group (ASO-miR-21 cells) as compared with the positive (A549/DDP cells) and the negative control (ASO-miR-NC cells) groups. (B) The flow cytometric results showed increased G2/M phase accumulation in the miR-21-silencing A549/DDP group (ASO-miR-21 cells) as compared with the positive (A549/DDP cells) and the negative control (ASO-miR-NC cells) groups. (C) Western blotting and quantitative polymerase chain reaction assay revealed reduced BCL-2 and CDK1 protein and mRNA expression levels in the miR-21-silencing A549/DDP group (ASO-miR-21 cells) as compared with the positive (A549/DDP cells) and the negative control (ASO-miR-NC cells) groups. The data are presented as the means \pm standard deviation. ${ }^{~} \mathrm{P}<0.05$, as compared with the parent group, ${ }^{*} \mathrm{P}<0.05$, as compared with the control group. $\mathrm{n}=5$. BCL-2, B-cell lymphoma 2; CDK1, cyclin-dependent kinase 1; miR-21, microRNA-21; ASO, antisense oligonucleotides; NC, negative control.

miR-21 silencing inhibits the tumor metastasis-related signaling pathway. The phosphoinositide 3-kinase (PI3K) signaling pathway is important in regulating cell apoptosis; AKT is an important molecule downstream of the PI3K signaling pathway. Therefore, the activation of AKT in the A549 cell groups was examined, by measuring the levels of phospho-AKT. Following 
A

Rhodamine 123
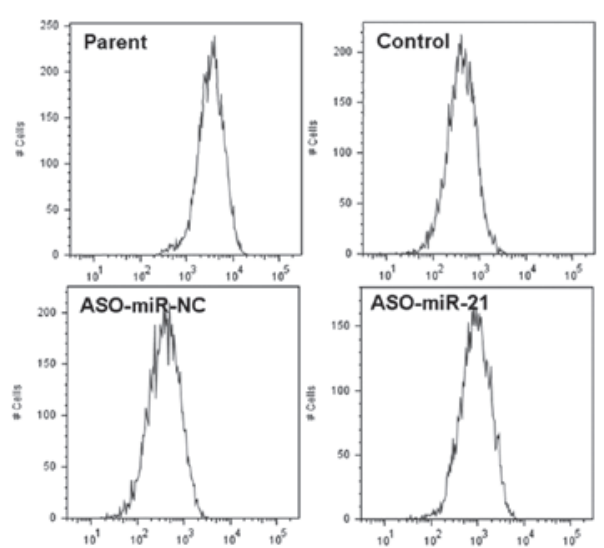

$\mathbf{B}$

\section{P-gp}
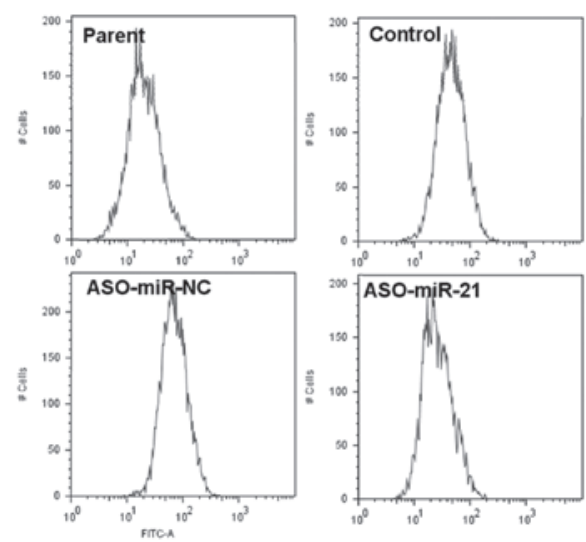
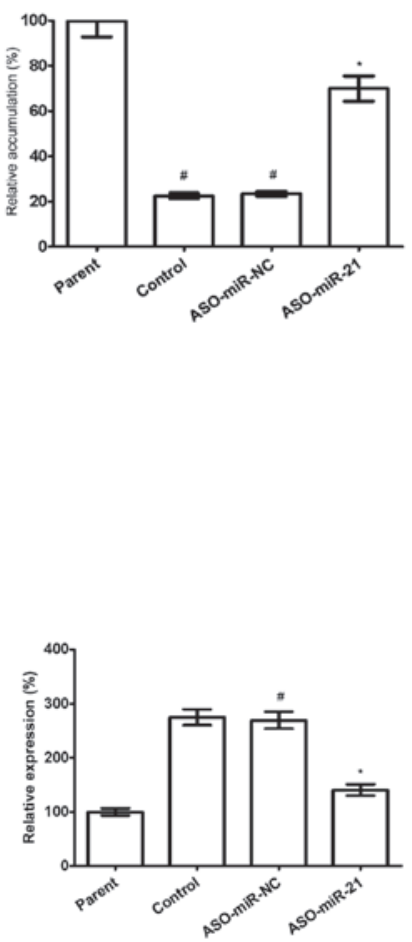

Figure 4. miR-21 increases cisplatin resistance by elevated drug excretion. (A) The results of the flow cytometry demonstrated an increase in Rhodamine 123 accumulation in the miR-21-silencing A549/DDP group (ASO-miR-21 cells) as compared with the positive (A549/DDP cells) and the negative control (ASO-miR-NC cells) groups. The data are presented as the means \pm standard deviation. ${ }^{*} \mathrm{P}<0.05$, as compared with the parent group, ${ }^{*} \mathrm{P}<0.05$, as compared with the control group. $\mathrm{n}=3$. (B) The results of the flow cytometry showed a reduction in P-gp expression levels in the miR-21-silencing A549/DDP group (ASO-miR-21 cells) in comparison with the positive (A549/DDP cells) and the negative control (ASO-miR-NC cells) groups. The data are presented as the means \pm standard deviation. ${ }^{\#} \mathrm{P}<0.05$, compared with the parent group, ${ }^{*} \mathrm{P}<0.05$, compared with the control group. $\mathrm{n}=3$. miR-21, microRNA-21; ASO, antisense oligonucleotides; NC, negative control. FITC, fluorescein isothiocyanate.
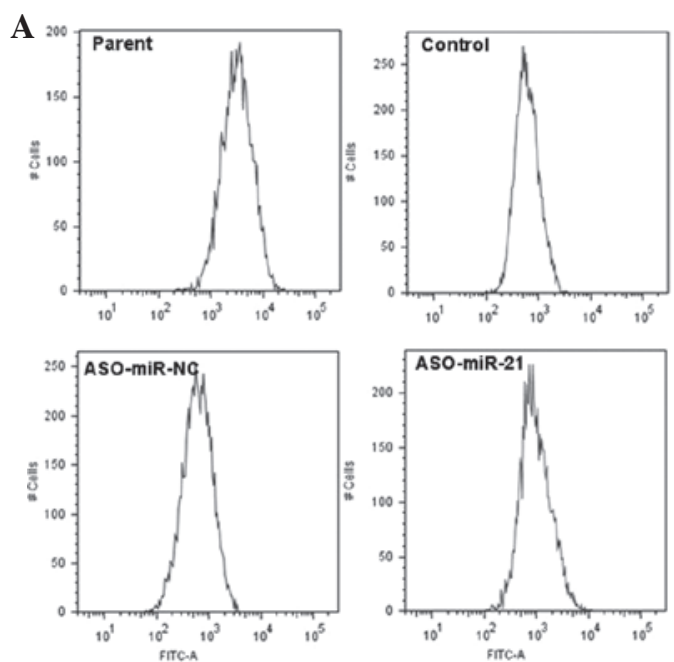

B
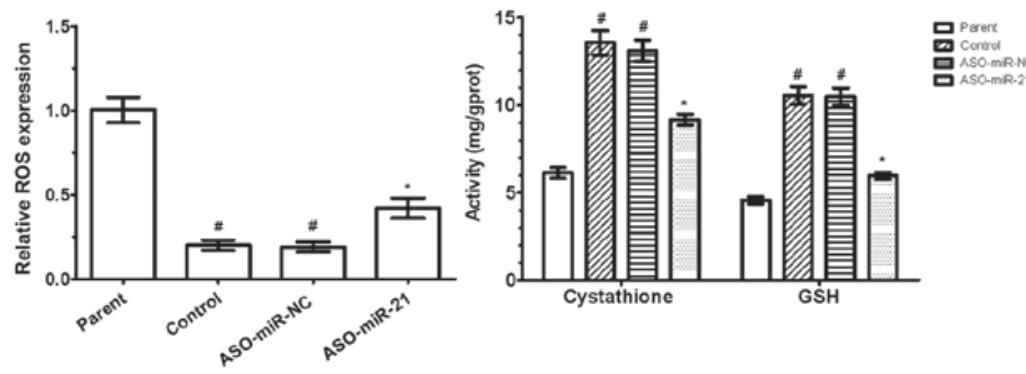

Figure 5. miR-21 increases cisplatin resistance by inhibiting cell oxidative damage metabolism. (A) The results of the flow cytometry showed increased expression levels of reactive oxygen species in the miR-21-silencing A549/DDP group (ASO-miR-21 cells) as compared with the positive (A549/DDP cells) and the negative control (ASO-miR-NC cells) groups. (B) Reduced cystathione and glutathione (GSH) expression levels were identified in the miR-21 silencing A549/DDP group (ASO-miR-21 cells) as compared with the positive (A549/DDP cells) and the negative control (ASO-miR-NC cells) groups. The data are presented as the means \pm standard deviation. ${ }^{\#} \mathrm{P}<0.05$, as compared with the parent group, $\mathrm{P}<0.05$, as compared with the control group. $\mathrm{n}=5$. miR-21, microRNA-21; ASO, antisense oligonucleotides; NC, negative control; FITC, fluorescein isothiocyanate; GSH, glutathione. 
A
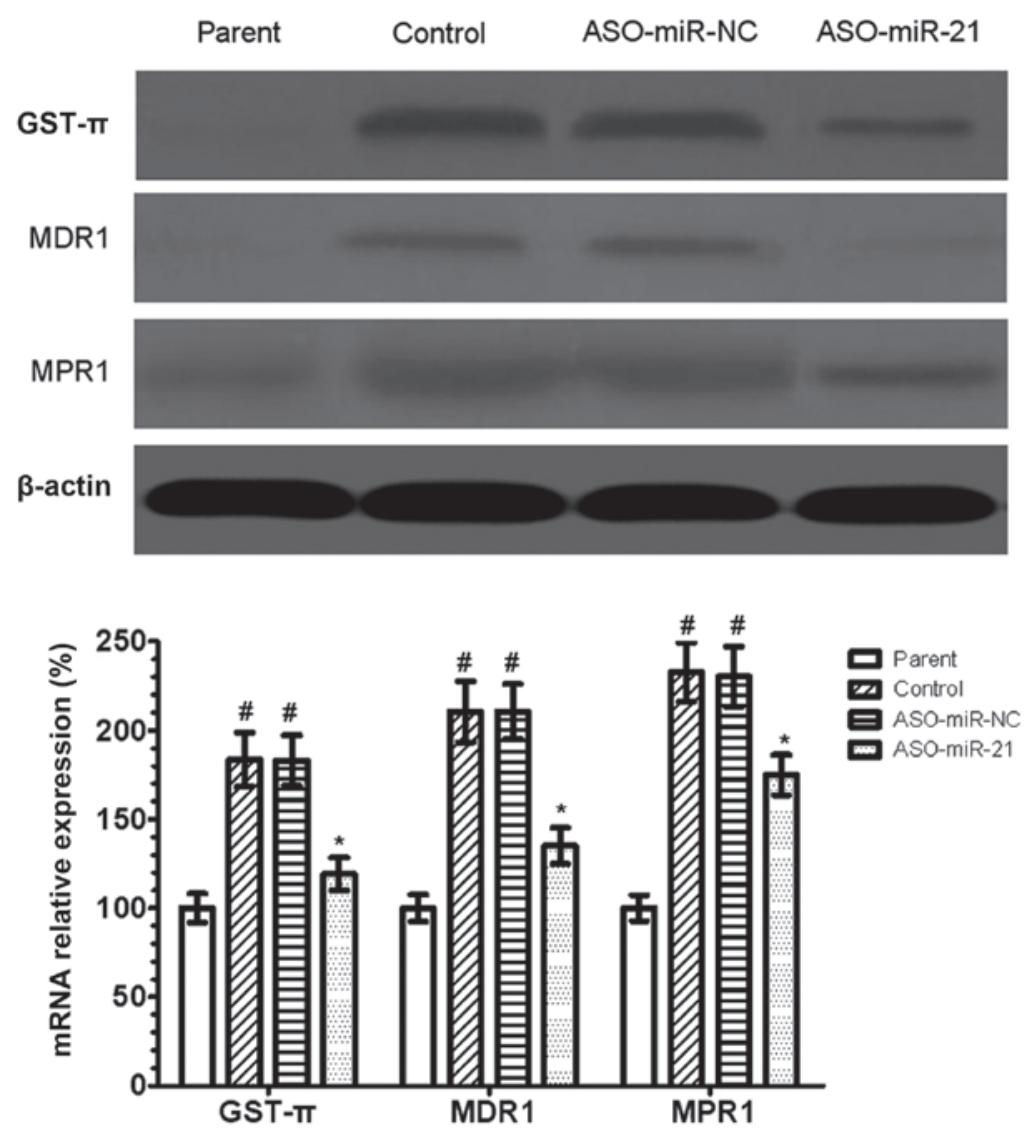

Figure 6. miR-21 enhances the mRNA and protein expression levels of multi-drug resistance (MDR) genes in cells. (A) Western blotting revealed reduced glutathione S-transferase- $\pi$ (GST- $\pi$ ), MDR1 and MPR1 protein expression levels in the miR-21-silencing A549/DDP group (ASO-miR-21 cells) as compared with the positive (A549/DDP cells) and the negative control (ASO-miR-NC cells) groups. $\beta$-actin served as an internal control for loading. (B) Quantitative polymerase chain reaction demonstrated reduced GST- $\pi$, MDR1 and MPR1 mRNA expression levels in the miR-21-silencing A549/DDP group (ASO-miR-21 cells) as compared with the positive (A549/DDP cells) and the negative control (ASO-miR-NC cells) groups. GAPDH served as an internal control for loading. The data are presented as the means \pm standard deviation. ${ }^{\#} \mathrm{P}<0.05$, as compared with the parent group, ${ }^{*} \mathrm{P}<0.05$ as compared with the control group. $\mathrm{n}=5$. miR-21, microRNA-21; ASO, antisense oligonucleotides; NC, negative control.

A

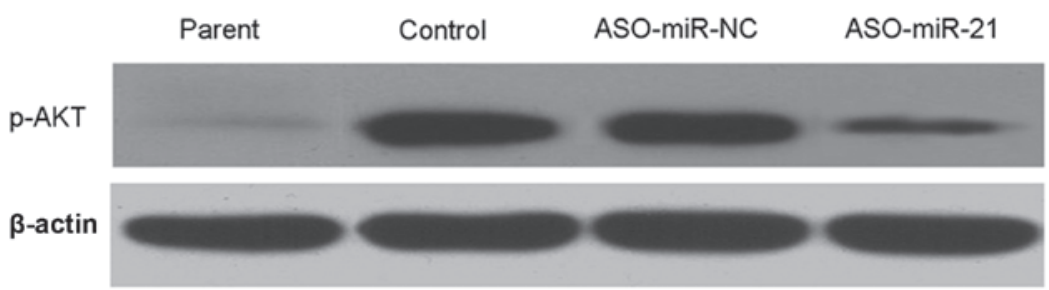

B

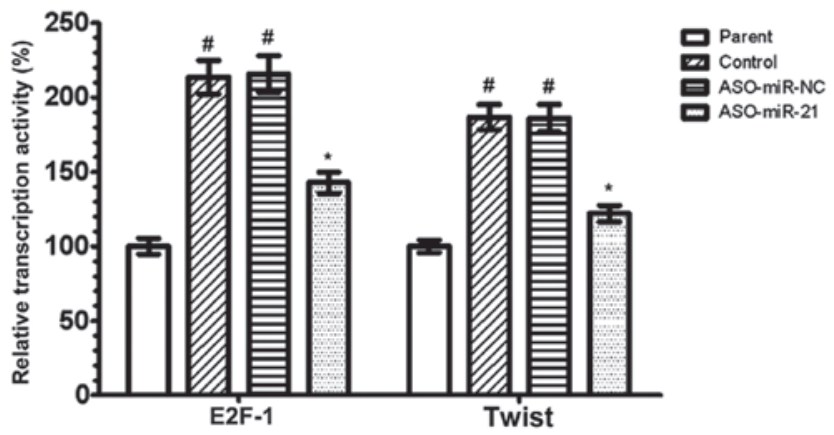

Figure 7. Effect of miR-21 on the tumor metastasis-related signaling pathway. (A) Western blotting revealed reduced AKT phosphorylation in the miR-21-silencing A549/DDP group (ASO-miR-21 cells) as compared with the positive (A549/DDP cells) and the negative control (ASO-miR-NC cells) groups. $\beta$-actin served as an internal control for loading. (B) A reporter gene assay demonstrated reduced E2F-1 and Twist transcriptional activity in the miR-21-silencing A549/DDP group (ASO-miR-21 cells) as compared with the positive (A549/DDP cells) and the negative control (ASO-miR-NC cells) groups. The data are presented as the means \pm standard deviation. ${ }^{~} \mathrm{P}<0.05$, compared with the parent group, ${ }^{*} \mathrm{P}<0.05$, compared with the control group. $\mathrm{n}=5$. miR-21, microRNA-21; ASO, antisense oligonucleotides; $\mathrm{NC}$, negative control. 
knockdown of miR-21 expression, AKT activation in A549/DDP cells was significantly reduced (Fig. 7A). This indicates that miR-21 is key to enhancing the activation of AKT signaling pathway.

E2F-1 and Twist are two important transcription factors downstream of AKT, which regulate apoptosis. Reporter genes were used to detect the activity of these two transcription factors. The results revealed that the knockdown of miR-21 expression significantly reduced the activity of these two transcription factors $(\mathrm{P}<0.05$; Fig. 7B).

The above results suggest that miR-21 exerts an enhancing effect on the PI3K signaling pathway, particularly in AKT activation, as well as the transcriptional activity of E2F-1 and Twist.

\section{Discussion}

Lung cancer has the highest incidence and mortality rate of any type of cancer in males and females in China (21). Cisplatin is a common drug used in lung cancer treatment; the main mechanism of action is to form an additive compound with tumor cell DNA and in turn induce tumor apoptosis (22). However, the clinical application of cisplatin can result in the generation of tumor drug resistance (23). Therefore, although chemotherapeutics are widely used in lung cancer, chemoresistance remains a significant therapeutic obstacle. The involvement of miRNAs in chemoresistance is a relatively new concept, and there has been increasing research into this subject. A number of studies have indicated that miR-21 in involved in the development chemoresistance in certain tumors and that the overexpression of miR-21 may attenuate cell death, whereas silencing of miR-21 was reported to stimulate this process (24).

In the present study, the miR-21 expression levels in A549/DDP cisplatin-resistant lung cancer cell lines were observed to be significantly increased compared with those of non-resistant A549 cells. miR-21 has been determined, in previous studies, to be one of the most important miRNAs in influencing the occurrence and development of tumors. Therefore, miR-21 was hypothesized to be important in cisplatin resistance in lung cancer (25).

In the present study, consistent with this hypothesis, following knockdown of miR-21 expression in A549/DDP cells, the cisplatin inhibition rate was significantly increased. This indicates that miR-21 enhances the cisplatin resistance in tumor cells. The apoptotic rate, as detected by Annexin V staining, was observed to be greatly increased and the cell BCL-2 expression level was reduced in the miR-21 silencing A549/DDP cells. Furthermore, miR-21 was shown to block the cell cycle at the G0/G1 phase and CDK1 expression levels were reduced when miR was knocked down. These results demonstrated that miR-21 produced an inhibitory effect on cisplatin-induced apoptosis.

Cisplatin resistance may be caused by various mechanisms, such as a reduction in intracellular drug concentration, the activation of detoxifying systems, the activation of DNA repair and the inhibition of apoptosis. In the present study, miR-21 was observed to inhibit cisplatin-induced apoptosis in tumor cells through three approaches: Enhancing drug efflux, promoting cisplatin inactivation and inhibiting the apoptotic signaling pathway.
MDR1 and MPR1 are important tumor MRD genes, which enhance the efflux of anticancer drugs. In the present study, miR-21 was found to increase the expression levels of these proteins, indicating that miR-21 inhibits the anticancer effect of cisplatin by enhancing the drug efflux from cells. Simultaneously, miR-21 also enhanced the expression levels of GST- $\pi$, cystathione and GSH cellular detoxification enzymes (26). The reduced expression levels of GST- $\pi$, cystathione and GSH following miR-21 knockdown demonstrated that cell detoxification was also an important mechanism of miR-21 for inhibiting the anticancer effect of cisplatin.

miR-21 was also observed to enhance the activation of the PI3K signaling pathway by increasing AKT activation and activating the transcription factors E2F-1 and Twist $(27,28)$. The PI3K signaling pathway is important in the inhibition of apoptosis (29). These findings demonstrate that miR-21 induces cisplatin resistance by inhibiting apoptotic signals.

In conclusion, the present study revealed that miR-21 is important in lung cancer cisplatin resistance. In the cisplatin-resistant tumor cells, the expression levels of miR-21 were significantly increased. miR-21 was observed to exert effects mainly by inhibiting cisplatin-induced apoptosis. miR-21 achieves the drug resistance effect through three mechanisms: Increasing MDR1 and MPR1 expression levels, and enhancing drug efflux from the cells; increasing GSH, superoxide dismutase and GST- $\pi$ expression levels and promoting drug inactivation; and inhibiting the PI3K signaling pathway and in turn inhibiting apoptotic signaling. These results suggest that miR-21 is key in lung cancer cisplatin resistance. The inhibition of miR-21 expression and the subsequent downstream functions may become a novel target in drug resistance therapy.

\section{References}

1. Lee RC and Ampros V: An extensive class of small RNAs in Caenorhabditis elgans. Science 294: 862-864, 2001.

2. Tan YG, Zhang YF, Guo CJ, Yang M and Chen MY: Screening of differentially expressed microRNA in ulcerative colitis related colorectal cancer. Asian Pac J Trop Med 6: 972-976, 2013.

3. Garg M,Potter JA and Abrahams VM: Identification of microRNAs That Regulate TLR2-Mediated Trophoblast Apoptosis and Inhibition of IL-6 mRNA. PLoS One 8: e77249, 2013.

4. Okoye IS, Coomes SM, Pelly VS, Czieso S, Papayannopoulos V, Tolmachova T,SeabraMC and Wilson MS: MicroRNA-containing T-regulatory-cell-derived exosomes suppress pathogenic T helper 1 cells. Immunity 41: 89-103, 2014.

5. Dang Z, Xu WH, Lu P, Wu N, Liu J, Ruan B, Zhou L, Song WJ and Dou KF: MicroRNA-135a inhibits cell proliferation by targeting Bmil in pancreatic ductal adenocarcinoma. Int J Biol Sci 10: 733-745, 2014

6. Wali RK, Hensing TA, Ray DW, Dela Cruz M, Tiwari AK, Radosevich A, Jepeal L, Fernando HC, Litle VR, Charlot M, et al: Buccal microRNA dysregulation in lung field carcinogenesis: gender-specific implications. Int J Oncol 45: 1209-1215, 2014

7. Lee KH, Lin FC, Hsu TI, Lin JT, Guo JH, Tsai CH, Lee YC, Lee YC, Chen CL, Hsiao M and Lu PJ: MicroRNA-296-5p (miR-296-5p) functions as a tumor suppressor in prostate cancer by directly targeting Pin1. Biochim Biophys Acta 1843: 2055-2066, 2014.

8. Xu G, Zhang Y, Wei J, et al: MicroRNA-21 promotes hepatocellular carcinoma HepG2 cell proliferation through repression of mitogen-activated protein kinase-kinase 3. BMC Cancer 13: 469, 2013.

9. Carthew RW and Sontheimer EJ: Origins and Mechanisms of miRNAs and siRNAs. Cell 136: 642-655, 2009. 
10. Zhou X, Wang X, Huang Z, Wang J, Zhu W, Shu Y and Liu P. Prognostic value of miR-21 in various cancers: an updating meta-analysis. PLoS One 9: e102413, 2014.

11. Li T, Leong MH, Harms B, Kennedy $G$ and Chen L: MicroRNA-21 as a potential colon and rectal cancer biomarker. World J Gastroenterol 19: 5615-5621, 2013.

12. Kadera BE, Li L, Toste PA, et al: MicroRNA-21 in pancreatic ductal adenocarcinoma tumor-associated fibroblasts promotes metastasis. PLoS One 8: e71978, 2013.

13. Asangani IA, Rasheed SA, Nikolova DA, et al: MicroRNA-21 (miR-21) post-transcriptionally downregulates tumor suppressor Pdcd4 and stimulates invasion, intravasation and metastasis in colorectal cancer. Oncogene 27: 2128-2136, 2008.

14. Zhu S, Wu H, Wu F, et al: MicroRNA-21 targets tumor suppressor genes in invasion and metastasis. Cell Res 18: 350-359, 2008

15. Kapoor S: microRNA-21 and its emerging role in tumor progression in systemic malignancies. Onkologie 36: 451, 2013.

16. Zhu Y, Liu XJ, Yang P, et al: Alkylglyceronephosphate synthase (AGPS) alters lipid signaling pathways and supports chemotherapy resistance of glioma and hepatic carcinoma cell lines Asian Pac J Cancer Prev 15: 3219-3226, 2014.

17. Yang T, Zheng ZM, Li XN, et al: MiR-223 modulates multidrug resistance via downregulation of ABCB1 in hepatocellular carcinoma cells. Exp Biol Med (Maywood) 238: 1024-1032, 2013.

18. Zeng X, Morgenstern R and Nyström AM: Nanoparticle-directed sub-cellular localization of doxorubicin and the sensitization breast cancer cells by circumventing GST-mediated drug resistance. Biomaterials 35: 1227-1239, 2014.

19. Chen J, Ding Z, Peng Y, Pan F, et al: HIF-1 $\alpha$ inhibition reverses multidrug resistance in colon cancer cells via downregulation of MDR1/P-glycoprotein. PLoS One 9: e98882, 2014.

20. Zhang N, Zhang Z, Wong IL, et al: 4,5-Di-substituted benzylimidazol-2-substituted amines as the structure template for the design and synthesis of reversal agents against P-gp-mediated multidrug resistance breast cancer cells. Eur J Med Chem 18: 74-83, 2014.
21. Siegel R, Naishadham D and Jemal A: Cancer statistics, 2013. CA Cancer J Clin 63: 11-30, 2013.

22. Panchuk R, Skorokhyd N, Chumak V, Lehka L, Omelyanchik S, Gurinovich V, Moiseenok A, Heffeter P, Berger W and Stoika R: Specific antioxidant compounds differentially modulate cy totoxic activity of doxorubicin and cisplatin: in vitro and in vivo study. Croat Med J 55: 206-217, 2014.

23. Jiao X, Zhao L, Ma M, et al: MiR-181a enhances drug sensitivity in mitoxantone-resistant breast cancer cells by targeting breast cancer resistance protein (BCRP/ABCG2). Breast Cancer Res Treat 139: 717-730, 2013.

24. Seca H, Lima RT, Lopes-Rodrigues V, Guimaraes JE, Almeida GM and Vasconcelos MH: Targeting miR-21 induces autophagy and chemosensitivity of leukemia cells. Curr Drug Targets 14: 1135-1143, 2013

25. Ren W, Wang X, Gao L, Li S, Yan X, Zhang J, Huang C, Zhang Y and Zhi K: MiR-21 modulates chemosensitivity of tongue squamous cell carcinoma cells to cisplatin by targeting PDCD4. Mol Cell Biochem 390: 253-262, 2014.

26. von Stechow L, Ruiz-Aracama A, van de Water B, et al: Identification of Cisplatin-Regulated Metabolic Pathways in Pluripotent Stem Cells. PLoS One 8: e76476, 2013.

27. Zheng S, Moehlenbrink J, Lu YC, et al: Arginine Methylation-Dependent Reader-Writer Interplay Governs Growth Control by E2F-1. Mol Cell 52: 37-51, 2013.

28. Hu P, Yang J, Hou Y, et al: LncRNA expression signatures of twist-induced epithelial-to-mesenchymal transition in MCF10A cells. Cell Signal 26: 83-93, 2014.

29. Chan JK, Blansit K, Kiet T, Sherman A, Wong G, Earle C and Bourguignon LY: The inhibition of miR-21 promotes apoptosis and chemosensitivity in ovarian cancer. Gynecol Oncol 132: 739-744, 2014. 